\title{
Sous-groupes additifs de rangs dénombrables dans un corps séparablement clos
}

\author{
Thomas Blossier
}

25 novembre 2010

\begin{abstract}
Résumé
Pour tout entier $n$, on construit des sous-groupes, infiniment définissables de rang de Lascar $\omega^{n}$, du groupe additif d'un corps séparablement clos.
\end{abstract}

\section{Introduction}

L'étude de certains groupes infiniment définissables dans les corps séparablement clos joue un rôle important dans la preuve de la conjecture de Mordell-Lang en caractéristique positive [10]. Dans la démonstration du théorème de Mordell-Lang pour les modules de Drinfeld, c'est l'étude de la structure «modulaire» de certains sous-groupes additifs qui est utilisée [9]. La classe des sous-groupes additifs est par-elle même intéressante, car très riche dans le contexte de la stabilité. Il est possible, en particulier, de construire des sous-groupes additifs de «rangs petits» avec certaines propriétés : par exemple une famille de groupes minimaux $\omega$-catégoriques deux à deux orthogonaux, des groupes minimaux avec des structures modulaires particulières, des groupes minimaux «minces non très minces» (voir [1], [2]). On peut également construire des groupes rangés de rangs infinis. En fait, dans [1], des sous-groupes additifs de rang $\omega$ sont construits. Une construction de types triviaux de rang $\omega$ avait été réalisée auparavant dans l'article [6] et ses auteurs remarquaient alors qu'il devait être possible d'obtenir des types d'autres rangs dénombrables par des constructions analogues. La généralisation de ces constructions s'avère techniquement assez complexe. L'objet du présent papier est d'expliciter, pour tout entier $n$, des constructions de sous-groupes additifs de rang dénombrable $\omega^{n}$. Notons qu'à notre connaissance, on ne sait pas pour l'instant construire un type de rang dénombrable $\omega^{\omega}$ dans un corps séparablement clos.

Pour toute la suite, nous fixons un corps $L$ séparablement clos non algébriquement clos de caractéristique $p$. On appelle sous-groupe additif, un sous-groupe de $(L,+)$ infiniment définissable dans $L$. Nous généralisons en fait la construction de sous-groupes additifs de rang $\omega$ pour obtenir :

1. Pour tout ordinal $\lambda<\omega^{\omega}$, une construction de sous-groupes additifs non monobasés de rang $\mathrm{U}$ égal à $\lambda$ (voir Corollaire 28). (Notons que $\omega^{\omega}$ désigne l'ordinal $\omega$

Mathematics Subject Classification : 03C45, 03C60, 12L12 
à la puissance $\omega$, en tant qu'exponentielle ordinale. En particulier $\omega^{\omega}$ est dénombrable.)

2. Pour toute famille dénombrable $\left(\lambda_{i}\right)_{i \in \omega}$ d'ordinaux strictement inférieurs à $\omega^{\omega}$, une construction de familles de sous-groupes additifs monobasés $G_{i}$ deux à deux orthogonaux et de rang $\mathrm{U}$ respectif égal à $\lambda_{i}$ (voir Corollaire 32 ).

Rappelons très rapidement certains résultats bien connus sur les corps séparablement clos : la théorie des corps séparablement clos de caractéristique $p$ et de degré d'imperfection $\nu$ est complète, pour chaque $p$ et $\nu$ fixés [8]. Pour obtenir l'élimination des quantificateurs, il suffit d'ajouter au langage, des fonctions appelées $\lambda$-fonctions (voir [4] ou [5]). On renvoie à l'introduction de [7] pour les notations et définitions concernant les $\lambda$-fonctions, mais elles ne seront pas nécessaires pour la lecture de nos constructions.

La clôture définissable de $A$, notée $\operatorname{dcl}(A)$, est le plus petit sous-corps de $L$ contenant $A$ et clos par les $\lambda$-fonctions et la clôture algébrique de $A$, notée acl $(A)$, est la clôture séparable de $A$.

Nous fixons $K$ une sous-structure élémentaire de $L$ et nous supposons que $L$ est $|K|^{+}$-saturé. Par élimination des quantificateurs, le type d'un uple $\bar{a}$ sur $K$ est déterminé par la donnée des équations algébriques satisfaites par une énumération des points de $\operatorname{dcl}(K, \bar{a})$ en fonction des points de $(K, \bar{a})$. Nous noterons $K\langle A\rangle$ le modèle premier au-dessus de $A \cup K$ qui est égal à acl $(K, A)$.

Fixons $b \in K \backslash K^{p}$. Afin de simplifier les notations, nous ne considérerons par la suite que des éléments ayant des arbres binaires de composantes (sur $b$ ) : soit $x$ un élément de $L$,

1. $x$ a un arbre binaire d'hauteur 1 si $x=x_{0}^{p}+b x_{1}^{p}$ avec $x_{0}$ et $x_{1}$ dans $L$. On appelle alors $x_{0}$ et $x_{1}$ les composantes de niveau 1 de $x$ et on note $x_{=1}$ l'uple $x_{0} x_{1}$.

2. $x$ a un arbre binaire d'hauteur $n+1$ si $x=x_{0}^{p}+b x_{1}^{p}$ avec $x_{0}$ et $x_{1}$ dans $L$ ayant des arbres binaires d'hauteur $n$. On appelle alors composantes de niveau $n+1$ de $x$, l'ensemble des composantes de niveau $n$ de $x_{0}$ et de $x_{1}$. On note $x_{=n+1}$ la concaténation de $x_{0=n}$ avec $x_{1=n}$.

3. $x$ a un arbre binaire d'hauteur infinie si $x$ a un arbre binaire d'hauteur $n$ pour tout $n$. On note alors $x_{\infty}$ l'uple de ses composantes.

On représente les arbres binaires de la manière suivante :

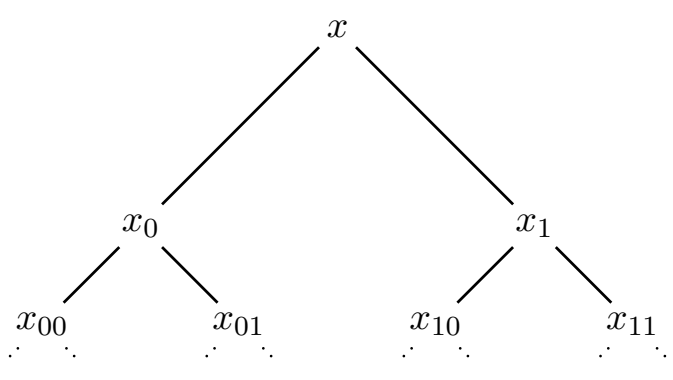

Remarque. L'ensemble $\mathcal{B}_{n}$ des éléments de $L$ ayant un arbre binaire d'hauteur $n$ (sur $b$ ) est un sous-groupe additif définissable $(\operatorname{sur} b)$. On notera $\mathcal{B}_{\infty}$ le sous-groupe additif 
infiniment définissable constitué des éléments de $L$ ayant un arbre binaire d'hauteur infinie. Remarquons que par élimination des quantificateurs, le type sur $K$ d'un élément $a$ de $\mathcal{B}_{\infty}$ est déterminé par le type d'isomorphisme de $K\left(a_{\infty}\right)$ dans le langage de pur corps. Notons qu'un élément $a$ dans $\mathcal{B}_{n}$ est interdéfinisable avec l'uple $a_{=n}$ au-dessus de $b$. Quand nous ne considérerons qu'une partie des composantes de niveau $n$ de $a$ dans $\mathcal{B}_{n}$, nous utiliserons la notation $a_{I}$ pour $I \subset[=n]$, où $[=n]$ désigne l'ensemble des indices permettant d'énumérer les composantes de niveau $n$. Nous utiliserons les mêmes notations pour un $k$-uple $a$ d'éléments de $\mathcal{B}_{n}$, par exemple $a_{I}$ pour une partie des composantes de niveau $n$ de $a$ pour $I \subset k \times[=n]$.

Nous allons par la suite construire des types dans $\mathcal{B}_{\infty}$ en utilisant uniquement des équations additives (c'est-à-dire des équations définies par des polynômes additifs ou $p$-polynômes) sur les composantes $x_{\infty}$. Les types additifs obtenus seront alors des génériques de sous-groupes additifs connexes (voir [3, Definition 2.5 et Lemma 2.6]).

On notera $X_{=n}$, respectivement $X_{\infty}$ les indéterminés des polynômes additifs portant sur les composantes $x_{=n}$, respectivement $x_{\infty}$. On notera RU le rang $U$ et $\mathrm{RT}$ le rang de transcendance. (Rappelons que $\mathrm{RT}(x ; K)$ est par définition le degré de transcendance de $K\langle x\rangle$ sur $K$.)

Dans la section 2, nous commençons par définir un type de relations entre les composantes, que nous appelons maillage, et qui nous permet selon la complexité $l$ de ces maillages, d'obtenir, dans la section 3, des sous-groupes additifs avec rang U supérieur ou égal à $\omega^{l}$ et ensuite, dans la section 4 , de majorer ce rang par $\omega^{l}$ en utilisant le même type de procédé que pour la construction de sous-groupes additifs de rang $\omega$ dans [1].

\section{Maillage}

Pour $k>0$, on note un $k$-uple $a, a=\left(a_{1}, \ldots, a_{k}\right)$ ou simplement $a=a_{1} \ldots a_{k}$.

Définition 1. On définit par induction sur $l \geq 1$, les $\left(k_{1}, \ldots, k_{l}\right)$-uples :

(i) Un $(k)$-uple est un $k$-uple d'éléments de $L$.

(ii) Un $\left(k_{1}, \ldots, k_{l+1}\right)$-uple est un $k_{1}$-uple de $\left(k_{2}, \ldots, k_{l+1}\right)$-uples.

La complexité et la décomposition d'un $\left(k_{1}, \ldots, k_{l}\right)$-uple sont respectivement $l$ et $\left(k_{1}, \ldots, k_{l}\right)$. On dira également que la complexité d'une décomposition $u=\left(k_{1}, . ., k_{l}\right)$ est $l$ et on définit $s(u):=k_{1} k_{2} \cdots k_{l}$.

\section{Remarque.}

(i) On se permet, si besoin, de regarder un (4,3)-uple, comme un (12)-uple, un $(3,4)$-uple, ou un $(2,3,2)$-uple. De manière générale un uple $a$ de décomposition $u$ peut aussi être regardé comme un $s(u)$-uple d'éléments de $L$, et $b \in a$ signifie que $b$ est un de ces $s(u)$ éléments.

(ii) Pour simplifier les notations, si $u=\left(k_{1}, \ldots, k_{l}\right)$ et $v=\left(k_{1}^{\prime}, \ldots, k_{l^{\prime}}^{\prime}\right)$ sont deux décompositions, la décomposition $\left(k_{1}, \ldots, k_{l}, k_{1}^{\prime}, \ldots, k_{l^{\prime}}^{\prime}\right)$ se note indifféremment $(u, v)$, $\left(k_{1}, \ldots, k_{l}, v\right)$ ou $\left(u, k_{1}^{\prime}, \ldots, k_{l^{\prime}}^{\prime}\right)$.

Définition 2. On dit qu'une décomposition est paire si elle est de la forme $v=(2 k, u)$ où $u$ est une décomposition. On note dans ce cas $v / 2$ la décomposition $(k, u)$. Enfin 
si $x_{1}$ et $x_{2}$ sont deux uples de décomposition $(k, u)$, on regarde l'uple $x_{1} x_{2}$ comme un uple de décomposition $(2 k, u)$.

Définition 3. On définit par induction $\operatorname{sur} l \geq 1$ et $n \geq 1$, les maillages de complexité $l$ et de hauteur $n$, portant sur des couples $(x, y)$ d'uples de complexité $l$ et respectivement de décompositions $u$ et $v(u, n)$.

(i) Maillage de complexité 1 et de hauteur 1 :

$$
\mathrm{M}_{(k)}^{1}(x, y):=\bigwedge_{i=1}^{k}\left(x_{i}=y_{i}^{p}+b y_{i+1}^{p}\right)
$$

pour $x=\left(x_{1}, \ldots, x_{k}\right)$ un $(k)$-uple et $y=\left(y_{1}, \ldots, y_{k+1}\right)$ un $(k+1)$-uple. On pose $v((k), 1):=(k+1)$.

(ii) Maillage de complexité $l$ et de hauteur $n+1$ :

$$
\mathrm{M}_{u}^{n+1}(x, y):=\exists z \mathrm{M}_{u}^{1}(x, z) \wedge \mathrm{M}_{w}^{n}(z, y)
$$

où $x, y$, et $z$ sont des uples de complexité $l$ et respectivement de décompositions $u, v$ et $w$ tels que $w=v(u, 1)$ et $v=v(w, n)$. On pose $v(u, n+1):=v=v(v(u, 1), n)$.

(iii) Maillage de complexité $l+1$ et de hauteur 1 :

$$
\mathrm{M}_{\left(k, k^{\prime}, u\right)}^{1}(x, y):=\bigwedge_{i=1}^{k} \mathrm{M}_{\left(k^{\prime}, u\right)}^{k^{\prime}+2}\left(x_{i}, y_{i} y_{i+1}\right)
$$

où $x$ et $y$ sont des uples de complexité $l+1$ et respectivement de décomposition $\left(k, k^{\prime}, u\right)$ et $v\left(\left(k, k^{\prime}, u\right), 1\right):=\left(k+1, v\left(\left(k^{\prime}, u\right), k^{\prime}+2\right) / 2\right)$.

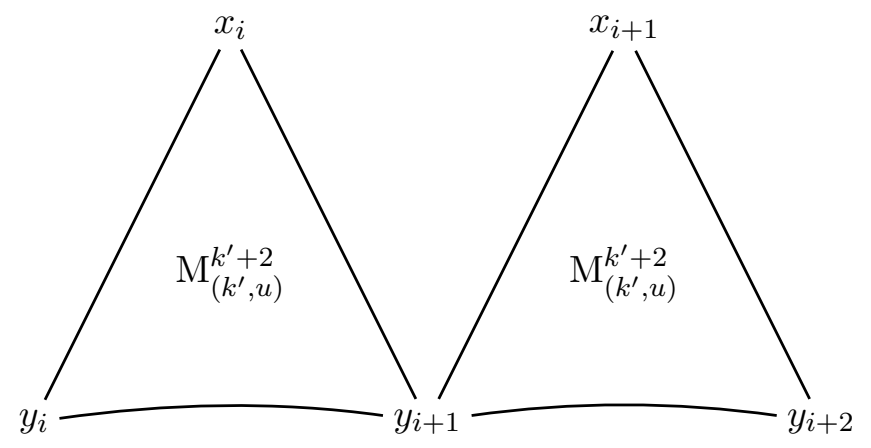

Remarque 4. On vérifie facilement que $v$ est bien définie, c'est-à-dire que pour tout $k^{\prime}$ et $u, v\left(\left(k^{\prime}, u\right), k^{\prime}+2\right)$ est paire. Pour cela on remarque par induction sur la complexité que si $u=\left(k_{1}, \ldots, k_{n}\right)$ et $v(u, 1)=\left(k_{1}^{\prime}, \ldots, k_{n}^{\prime}\right)$ alors $k_{1}^{\prime}=k_{1}+1$ et pour tout $i>1$, $k_{i}^{\prime}>k_{i}$. Notons que si $n \geq 2$ alors on a également la relation $k_{2}^{\prime}=k_{2}+1$.

La propriété suivante se vérifie également par simple induction. 
Propriété 5. Pour chaque décomposition u, il existe une suite strictement croissante $\left(m_{u, n}\right)_{n>0}$ d'entiers strictement positifs, une suite $\left(I_{u, n}\right)_{n>0}$ où $I_{u, n} \subset s(u) \times\left[=m_{u, n}\right]$, et pour chaque $n>0$, un système $S_{u, n}$ d'égalités entre les variables appartenant à l'uple $X_{=m_{u, n}}$ où $X$ est un uple de $s(u)$ indéterminées, telles que pour tout uple $x \in \mathcal{B}_{\infty}^{\times s(u)}$ de décomposition $u$,

(i) $\exists y \mathrm{M}_{u}^{n}(x, y) \leftrightarrow S_{u, n}(x)$

(ici $S_{u, n}(x)$ signifie que l'uple $x_{=m_{u, n}}$ satisfait le système $S_{u, n}$ );

(ii) si $S_{u, n}(x)$ alors :

- $x_{I_{u, n}}$ est l'unique y tel que $\mathrm{M}_{u}^{n}(x, y)$;

- pour tout $j \in s(u) \times\left[=m_{u, n}\right]$, il existe $i \in I_{u, n}$ tel que $x_{j}=x_{i}$, en particulier $x$ et $x_{I_{u, n}}$ sont interdéfinissables.

\section{Grills de complexité arbitraire}

Nous donnons dans cette section une construction de types de rang U supérieur ou égal à $\omega^{l}$. On utilise pour cette construction un maillage entre les composantes qui nous permettra dans la section suivante de compléter cette construction afin d'obtenir des types de rang $\mathrm{U}$ égal à $\omega^{l}$.

Pour la suite, fixons une décomposition $u$ de complexité $l$.

Définition 6. On définit le grill de décomposition $u$ par

$$
\operatorname{Grill}_{u}:=x \in \mathcal{B}_{\infty}^{\times s(u)} \wedge \bigwedge_{n=1}^{\infty} S_{u, n}(x)
$$

où $\left(S_{u, n}\right)_{n>0}$ est la famille de systèmes d'égalités entre certaines composantes vue dans la Propriété 5.

Lemme 7. Le grill de décomposition u est un sous-groupe additif de $\mathcal{B}_{\infty}^{\times s(u)}$, infiniment définissable sur b et connexe.

Démonstration. Le fait que Grill $u$ est un sous-groupe additif infiniment définissable sur $b$ est évident puisque les systèmes $S_{u, n}$ correspondent à des égalités entre composantes. D'autre part, il est facile de vérifier que «x $\in$ Grill $_{u} »$ n'impose pour aucun $n>0$ de relations algébriques, au sens des corps, entre les éléments de $x_{I_{u, n}}$. Donc les équations $\ll x \in$ Grill $_{u} »$ et les inéquations «les éléments de $x_{I_{u, n}}$ sont algébriquement indépendants au-dessus de $K \gg$ pour tout $n>0$, détermine un type complet sur $K$, qui est alors l'unique générique de $\mathrm{Grill}_{u}$ sur $K$. Le groupe Grill ${ }_{u}$ est donc connexe.

On note par la suite $p_{u}$ le type générique de $\operatorname{Grill}_{u}$ au-dessus de $K$.

Nous allons montrer que Grill ${ }_{u}$ est de rang U supérieur ou égal à $\omega^{l}$. Pour cela, on utilise le fait que $p_{u}$ a un arbre de $p$-composantes qui est minoré au sens suivant.

Définition 8. Nous définissons par induction sur la complexité la notion d'arbre minoré sur des uples de $\mathcal{B}_{\infty}$ :

(i) Un uple $a$ de complexité 1 a un arbre minoré sur $K$ s'il existe deux suites strictement croissantes $\left(i_{n}\right)_{n<\omega}$ et $\left(k_{n}\right)_{n<\omega}$ d'entiers, et une suite d'uples $a^{n}$ de complexité 
1 et de décomposition $\left(k_{n}\right)$, telles que pour chaque $n$,

- $a^{n} \subset a_{=i_{n}}$;

- $a^{n}=\left(a_{1}^{n}, \ldots, a_{k_{n}}^{n}\right)$ tel que $a_{j}^{n} \notin K\left\langle a_{j+1}^{n}, \ldots, a_{k_{n}}^{n}\right\rangle$ pour $j \in\left\{1, \ldots, k_{n}\right\}$.

(ii) Un uple $a$ de complexité $l+1$ a un arbre minoré sur $K$ s'il existe deux suites strictement croissantes $\left(i_{n}\right)_{n<\omega}$ et $\left(k_{n}\right)_{n<\omega}$ d'entiers, et une suite d'uples $a^{n}$ de complexité $l+1$ et de décomposition $\left(k_{n}, v_{n}\right)$, telles que pour chaque $n$,

$-a^{n} \subset a_{=i_{n}}$;

- $a^{n}=\left(a_{1}^{n}, \ldots, a_{k_{n}}^{n}\right)$ tel que l'uple $a_{j}^{n}$ de complexité $l$ a un arbre minoré sur $K\left\langle a_{j+1}^{n}, \ldots, a_{k_{n}}^{n}\right\rangle$ pour tout $j \in\left\{1, \ldots, k_{n}\right\}$.

Remarque. Si un uple $a$ a un arbre minoré sur $K^{\prime} \supseteq K$ (où $K^{\prime}$ est une extension élémentaire de $K$ ) alors cet uple a un arbre minoré sur $K$.

Lemme 9. Si un uple a de complexité l a un arbre minoré sur $K$, alors $\operatorname{RU}(a / K) \geq \omega^{l}$.

Démonstration. Soient $\left(i_{n}\right)_{n<\omega}$ et $\left(k_{n}\right)_{n<\omega}$ deux suites strictement croissantes d'entiers, et $\left(a^{n}\right)_{n<\omega}$ une suite d'uples de complexité $l$ comme dans la définition ci-dessus.

La preuve se fait par induction sur $l$.

Si $l=1$, pour tout $n<\omega$,

$$
\mathrm{RU}(a / K) \geq \operatorname{RU}\left(a^{n} / K\right) \geq \sum_{j=1}^{k_{n}} \mathrm{RU}\left(a_{j}^{n} / K\left\langle a_{j+1}^{n}, \ldots, a_{k_{n}}^{n}\right\rangle\right) .
$$

Par hypothèse pour tout $j \in\left\{1, \ldots, k_{n}\right\}, \operatorname{RU}\left(a_{j}^{n} / K\left\langle a_{j+1}^{n}, \ldots, a_{k_{n}}^{n}\right\rangle\right) \geq 1$, donc $\mathrm{RU}(a / K) \geq$ $k_{n}$, ceci pour tout $n<\omega$. Par conséquent $\operatorname{RU}(a / K) \geq \omega$.

Si $l=l^{\prime}+1$, pour tout $n<\omega$,

$$
\mathrm{RU}(a / K) \geq \operatorname{RU}\left(a^{n} / K\right) \geq \sum_{j=1}^{k_{n}} \operatorname{RU}\left(a_{j}^{n} / K\left\langle a_{j+1}^{n}, \ldots, a_{k_{n}}^{n}\right\rangle\right) .
$$

Par hypothèse pour tout $j \in\left\{1, \ldots, k_{n}\right\}$, l'uple $a_{j}^{n}$ de complexité $l^{\prime}$ a un arbre minoré sur $K\left\langle a_{j+1}^{n}, \ldots, a_{k_{n}}^{n}\right\rangle$ et donc par hypothèse d'induction $\mathrm{RU}\left(a_{j}^{n} / K\left\langle a_{j+1}^{n}, \ldots, a_{k_{n}}^{n}\right\rangle\right) \geq \omega^{l^{\prime}}$. D'où pour tout $n<\omega, \operatorname{RU}(a / K) \geq \omega^{l^{\prime}} k_{n}$ et donc $\operatorname{RU}(a / K) \geq \omega^{l}$.

Étant donnés un maillage sur un couple $(a, b)$ et un sous-uple $a^{\prime}$ de $a$, nous utiliserons par la suite le sous-uple de $b$ constitué des composantes de $a^{\prime}$ dans $b$.

Définition 10. Soient $n>0, a$ un uple de décomposition $u$ et $b$ un uple de décomposition $v=v(u, n)$ tels que $M_{u}^{n}(a, b)$. Soient $\left(a_{1}, \ldots, a_{s(u)}\right)$ et $\left(b_{1}, \ldots, b_{s(v)}\right)$ les énumérations des uples $a$ et $b$. Pour $1 \leq i \leq s(u)$, on note $C_{u, i}^{n}$ l'ensemble des indices dans $\{1, \ldots, s(v)\}$ énumérant les éléments du $s(v)$-uple $b$ qui correspondent «naturellement» par le maillage à des composantes de $a_{i}$.

Cet ensemble ne dépend que de $u, i$ et $n$ et se définit de manière précise par induction sur la complexité de $u$ et la hauteur $n$ :

1. $C_{(k), i}^{1}=\{i, i+1\}$ pour $1 \leq i \leq k$,

2. $C_{u, i}^{n+1}=\cup_{j \in C_{u, i}^{n}} C_{v(u, n), j}^{1}$ pour $1 \leq i \leq s(u)$, 
3. $C_{\left(k, k^{\prime}, u\right),(i-1) \cdot s\left(\left(k^{\prime}, u\right)\right)+j}^{1}=(i-1) \cdot s\left(v\left(\left(k^{\prime}, u\right), k^{\prime}+2\right) / 2\right)+C_{\left(k^{\prime}, u\right), j}^{k^{\prime}+2}$ pour $1 \leq i \leq k$ et $1 \leq j \leq s\left(\left(k^{\prime}, u\right)\right)$.

Pour $1 \leq i \leq s(u)$, on dira que $x$ est une composante de $a_{i}$ au niveau de $b$ par rapport à $a$ si $x=b_{j}$ pour un $j \in C_{u, i}^{n}$.

(Pour $n=0$, on pose $C_{u, i}^{0}=\{i\}$. )

La propriété suivante se vérifie par induction sur la complexité et la hauteur du maillage.

Propriété 11. Soient $n>0, a=\left(a_{1}^{\prime}, \ldots, a_{k}^{\prime}\right)$ un uple de décomposition $u=\left(k, u^{\prime}\right)$ et $b=\left(b_{1}^{\prime}, \ldots, b_{k+n}^{\prime}\right)$ un uple de décomposition $v=v(u, n)=\left(k+n, v^{\prime}\right)$ tels que $M_{u}^{n}(a, b)$ (ici les $a_{i}^{\prime}$ sont des uples de décomposition $u^{\prime}$, ou des éléments si la complexité de a est 1). Si $x$ est un élément de a appartenant (ou, si a est de complexité 1 , égal) à $a_{j}^{\prime}$ alors l'une des composantes de $x$ au niveau de $b$ par rapport à a appartient (ou est égale) $\grave{a} b_{j}^{\prime}$ et aucune composante de $x$ n'appartient (ou n'est égale) à $b_{l}^{\prime}$ pour $1 \leq l<j$. Le maillage étant symétrique, on a la même propriété en considérant $a_{j}^{\prime}, b_{j+n}^{\prime}$ et $b_{l}^{\prime}$ pour $j+n<l \leq k+n$.

Lemme 12. Les ensembles $C_{u, i}^{n}$ sont des intervalles tels que pour tout $n \geq 1$,
1. $\min C_{u, 1}^{n}=1$ (et $\max C_{u, s(u)}^{n}=s(v(u, n))$ symétriquement);
2. $\min C_{u, i}^{n}<\min C_{u, i+1}^{n} \leq \max C_{u, i}^{n}<\max C_{u, i+1}^{n}$ pour tout $1 \leq i<s(u)$.

Démonstration. Le passage de la hauteur $n$ à la hauteur $n+1$ est immédiat. Supposons le résultat vérifié pour une décomposition $\left(k^{\prime}, u\right)$ et considérons la décomposition $\left(k, k^{\prime}, u\right)$. Rappelons que pour tout $1 \leq i \leq k$ et tout $1 \leq j \leq s\left(\left(k^{\prime}, u\right)\right)$,

$$
C_{\left(k, k^{\prime}, u\right),(i-1) \cdot s\left(\left(k^{\prime}, u\right)\right)+j}^{1}=(i-1) \cdot s\left(v\left(\left(k^{\prime}, u\right), k^{\prime}+2\right) / 2\right)+C_{\left(k^{\prime}, u\right), j}^{k^{\prime}+2} .
$$

Il suit par induction que les ensembles $C_{\left(k, k^{\prime}, u\right), l}^{1}$, pour $1 \leq l \leq s\left(\left(k, k^{\prime}, u\right)\right)$, sont des intervalles vérifiant la première propriété. Soit $1 \leq l<s\left(\left(k, k^{\prime}, u\right)\right)$ alors si $l=$ $(i-1) \cdot s\left(\left(k^{\prime}, u\right)\right)+j$ avec $1 \leq i \leq k$ et $j<s\left(\left(k^{\prime}, u\right)\right)$ la seconde propriété suit également par induction. Sinon $l+1=i \cdot s\left(\left(k^{\prime}, u\right)\right)+1$. Par la Propriété 11 et la première propriété de ce lemme on obtient,

$$
\begin{aligned}
\min C_{\left(k, k^{\prime}, u\right), l}^{1} & <i \cdot s\left(v\left(\left(k^{\prime}, u\right), k^{\prime}+2\right) / 2\right)+1=\min C_{\left(k, k^{\prime}, u\right), l+1}^{1} \\
& <(i+1) \cdot s\left(v\left(\left(k^{\prime}, u\right), k^{\prime}+2\right) / 2\right)=\max C_{\left(k, k^{\prime}, u\right), l}^{1} \\
& <\max C_{\left(k, k^{\prime}, u\right), l+1}^{1} .
\end{aligned}
$$

Définition 13. Soient $n>0, a$ un uple de décomposition $u$ et $b$ un uple de décomposition $v=v(u, n)$ tels que $M_{u}^{n}(a, b)$. Si $a^{\prime}=\left(a_{k}, \ldots, a_{l}\right)$ est un sous-uple d'éléments consécutifs de l'uple $a$, on note alors $a_{\operatorname{niv}(b / a)}^{\prime}$ le sous-uple de $b$ constitué des éléments de $b$ qui correspondent «naturellement» par le maillage à des composantes d'éléments de $a^{\prime}$. Plus précisément $a_{\text {niv }(b / a)}^{\prime}$ est le sous-uple $\left(b_{s}, \ldots, b_{t}\right)$ d'éléments consécutifs de $b$ tel que $s=\min C_{u, k}^{n}$ et $t=\max C_{u, l}^{n}$. Notons que cette opération est transitive dans le 
sens suivant : si $c$ est un uple de décomposition $w=v(u, n+m)$ tel que $M_{v}^{m}(b, c)$ (ou de manière équivalente tel que $\left.M_{u}^{n+m}(a, c)\right)$ alors

$$
a_{\mathrm{niv}(c / a)}^{\prime}=\left(a_{\operatorname{niv}(b / a)}^{\prime}\right)_{\operatorname{niv}(c / b)} .
$$

(Pour $n=0$, on pose $a_{\operatorname{niv}(a / a)}^{\prime}=a^{\prime}$.)

Définition 14. Pour la suite on appelle uple extrait $a^{\prime}$ d'un uple $a$ de complexité $l$, tout uple (ou élément) apparaissant dans la décomposition de $a$. Cette notion est définie par induction sur la complexité $l$ :

- si $l=1$ et $a=\left(a_{1}, \ldots, a_{k}\right)$ alors $a^{\prime}$ est un uple extrait de $a$ si et seulement si $a^{\prime}=a$ ou $a^{\prime}=a_{i}$ où $a_{i}$ est l'un des éléments de l'uple $a$. Dans le premier cas on dira que $a^{\prime}$ est un uple extrait de complexité 1 , dans le second un uple extrait de complexité 0 .

- si $a=\left(a_{1}, \ldots, a_{k}\right)$ est un $k$-uple $\left(a_{1}, \ldots, a_{k}\right)$ d'uples de complexité $l$ alors $a^{\prime}$ est un uple extrait de $a$ si et seulement si $a^{\prime}=a$ (dans ce cas $a^{\prime}$ est un uple extrait de complexité $l+1$ ) ou $a^{\prime}$ est un uple extrait (de compexité $l^{\prime} \leq l$ ) de l'un des $a_{i}$.

De plus si $a^{\prime}$ est un uple extrait de $a$ on note $a^{D\left(a^{\prime}\right)}$ l'uple des éléments de $a$ à droite de $a^{\prime}$, que l'on appelle reste à droite.

Lemme 15. Soient deux uples a et $b$ vérifiant $M_{u}^{n}(a, b)$ pour un entier $n \geq 1$ et la décomposition u de a. Considérons $a^{\prime}$ un uple extrait de a de complexité $l^{\prime}$.

Il existe alors un unique uple extrait $b^{\prime}$ de $b$ de même complexité $l^{\prime}$ qui soit un préfixe (sous-uple initial) de $a_{\operatorname{niv}(b / a)}^{\prime}$.

De plus $a_{\operatorname{niv}(b / a)}^{D\left(a^{\prime}\right)} \subseteq b^{D\left(b^{\prime}\right)}$ (ici $a_{\operatorname{niv}(b / a)}^{D\left(a^{\prime}\right)}$ dénote l'uple $\left(a^{D\left(a^{\prime}\right)}\right)_{\operatorname{niv}(b / a)}$ qui est le sousuple des éléments de $b$ correspondant par le maillage à des composantes de $\left.a^{D\left(a^{\prime}\right)}\right)$.

Enfin si $a^{\prime \prime}$ est un second uple extrait de a tel que $a^{\prime \prime} \subseteq a^{\prime}$ alors l'uple extrait $b^{\prime \prime}$ de $b$ correspondant à $a^{\prime \prime}$ vérifie $b^{\prime \prime} \subseteq b^{\prime}$.

Démonstration. La preuve se fait par induction sur $n$ et la complexité de $a$.

Si la complexité est 1 et $n=1$. Alors $a=\left(a_{1}, \ldots, a_{k}\right)$ et $b=\left(b_{1}, \ldots, b_{k+1}\right)$ tels que $a_{i}=b_{i}^{p}+b_{i+1}^{p}$. Si $a^{\prime}=a_{i}$ alors $b^{\prime}$ vaut nécessairement $b_{i}$. Dans ce cas, si $i<k$ on a $a^{D\left(a_{i}\right)}=a_{i+1} \ldots a_{k}$ et $a_{\operatorname{niv}(b / a)}^{D\left(a_{i}\right)}=b_{i+1} \ldots b_{k+1}=b^{D\left(b_{i}\right)} ;$ sinon on a $a^{D\left(a_{k}\right)}=\emptyset$ et $a_{\text {niv }(b / a)}^{D\left(a_{k}\right)}=\emptyset \subseteq b_{k+1}=b^{D\left(b_{k}\right)}$. Si $a^{\prime}=a$, alors $b^{\prime}=b$ et $a^{D(a)}=b^{D(b)}=\emptyset$. La dernière propriété est évidemment vérifiée.

Passage de $n$ à $n+1$ : soient $a, b$ vérifiant $M_{u}^{n+1}(a, b)$. Considérons $a^{\prime}$ un uple extrait de $a$ de complexité $l^{\prime}$. Alors il existe $c$ de décomposition $w(=v(u, 1))$ tel que $M_{u}^{1}(a, c)$ et $M_{w}^{n}(c, b)$. Par hypothèse d'induction, il existe un unique uple extrait $c^{\prime}$ de $c$ de complexité $l^{\prime}$ qui soit un préfixe de $a_{\operatorname{niv}(c / a)}^{\prime}$ et il existe un unique uple extrait $b^{\prime}$ de $b$ de complexité $l^{\prime}$ qui soit un préfixe $\operatorname{de} c_{\operatorname{niv}(b / c)}^{\prime}$. Mais alors $c_{\operatorname{niv}(b / c)}^{\prime}$ est un préfixe de $\left(a_{\operatorname{niv}(c / a)}^{\prime}\right)_{\operatorname{niv}(b / c)}=a_{\operatorname{niv}(b / a)}^{\prime}$ donc $b^{\prime}$ est également un préfixe de $a_{\operatorname{niv}(b / a)}^{\prime}$. De plus $a_{\text {niv }(c / a)}^{D\left(a^{\prime}\right)} \subseteq c^{D\left(c^{\prime}\right)}$ et $c_{\text {niv }(b / c)}^{D\left(c^{\prime}\right)} \subseteq b^{D\left(b^{\prime}\right)}$ d'où $a_{\text {niv }(b / a)}^{D\left(a^{\prime}\right)}=\left(a_{\operatorname{niv}(c / a)}^{D\left(a^{\prime}\right)}\right)_{\operatorname{niv}(b / c)} \subseteq b^{D\left(b^{\prime}\right)}$. Enfin si $a^{\prime \prime}$ est un second uple extrait de $a$ tel que $a^{\prime \prime} \subseteq a^{\prime}$, alors par hypothèse d'induction $c^{\prime \prime} \subseteq c^{\prime}$ et donc $b^{\prime \prime} \subseteq b^{\prime}$. 
Passage de la complexité $l$ à $l+1$ : soient $a, b$ de complexités $l+1$ vérifiant $M_{u}^{1}(a, b)$. Alors $u$ est de la forme $\left(k, r, u^{\prime}\right)$ et $a=\left(a_{1}, \ldots, a_{k}\right), b=\left(b_{1}, \ldots, b_{k+1}\right)$ avec $M_{\left(r, u^{\prime}\right)}^{r+2}\left(a_{i}, b_{i} b_{i+1}\right)$ pour tout $i \in\{1, \ldots k\}$. Soit $a^{\prime}$ un uple extrait de complexité $l^{\prime}$. Si $a^{\prime}=a$ (respectivement $a^{\prime}=a_{i}$ pour un $i$ ), alors $b^{\prime}=b$ (respectivement $b^{\prime}=b_{i}$ pour ce même $i$ ) et on vérifie facilement que $a_{\operatorname{niv}(b / a)}^{D\left(a^{\prime}\right)} \subseteq b^{D\left(b^{\prime}\right)}$. Sinon, $a^{\prime}$ est un uple extrait d'un $a_{i}$ de complexité $l^{\prime}<l$. Par hypothèse d'induction sur le couple $\left(a_{i}, b_{i} b_{i+1}\right)$ de deux uples de complexité $l$, il existe un unique uple extrait $b^{\prime}$ de $b_{i} b_{i+1}$ de complexité $l^{\prime}$ tel que $b^{\prime}$ est un préfixe de $a_{\text {niv }\left(b_{i} b_{i+1} / a_{i}\right)}^{\prime}$. Remarquons que l'uple $a_{\text {niv }\left(b_{i} b_{i+1} / a_{i}\right)}^{\prime}$ (où $a^{\prime}$ est considéré comme uple extrait de $a_{i}$ ) est égal à $a_{\text {niv }(b / a)}^{\prime}$ (où $a^{\prime}$ est considéré comme uple extrait de $a$ ). De plus comme $l^{\prime}<l$, l'uple $b^{\prime}$ est nécessairement un uple extrait de $b$. Notons enfin que $a_{i}=\left(a_{i, 1}, \ldots, a_{i, r}\right)$ et $b_{i} b_{i+1}=\left(b_{i, 1}, \ldots, b_{i, r}, b_{i, r+1}, b_{i+1,1}, \ldots, b_{i+1, r}, b_{i+1, r+1}\right)$ et qu'en particulier si $a^{\prime}=a_{i, s}$ alors $b^{\prime}=b_{i, s}$ par induction. Il suit que quel que soit l'uple extrait $a^{\prime}$ de $a_{i}$ de complexité $l^{\prime}<l$, on a $b^{\prime} \subseteq b_{i}$. Alors par induction, $\left(a_{i}\right)_{\operatorname{niv}\left(b_{i} b_{i+1}\right)}^{D\left(a^{\prime}\right.} \subseteq b_{i}^{D\left(b^{\prime}\right)} b_{i+1}$. Il suit que $a_{\operatorname{niv}(b / a)}^{D\left(a^{\prime}\right)} \subseteq b^{D\left(b^{\prime}\right)}=b_{i}^{D\left(b^{\prime}\right)} b_{i+1} \ldots b_{k+1}$ car $a^{D\left(a^{\prime}\right)}=a_{i}^{D\left(a^{\prime}\right)} a_{i+1} \ldots a_{k}$ et $\left(a_{j}\right)_{\operatorname{niv}(b / a)}=b_{j} b_{j+1}$ pour tout $j$.

Proposition 16. RU $\left(\right.$ Grill $\left._{u}\right) \geq \omega^{l}$.

Démonstration. Soit $x$ une réalisation de $p_{u}$. Montrons que pour tout uple extrait $a^{\prime}$ de complexité non nul d'un uple $a$ tel que $M_{u}^{m}(x, a)$ pour un entier $m, a^{\prime}$ a un arbre minoré sur $K\left\langle a^{D\left(a^{\prime}\right)}\right\rangle$. En particulier on en déduit que $x$ a un arbre minoré sur $K$ et donc que $\operatorname{RU}(x / K) \geq \omega^{l}$.

Nous montrons ce résultat par induction sur la complexité $l^{\prime}$ de $a^{\prime}$. Pour tout entier $n$, notons $a^{n}$ tel que $M_{v}^{n}\left(a, a^{n}\right), a^{\prime n} l^{\prime}$ uple extrait de $a^{n}$ correspondant à $a^{\prime}$ via le lemme précédent, et enfin $a^{D, n}$ le reste à droite de $a^{\prime n}$ dans $a^{n}$. Le lemme précédent implique que $a_{\infty}^{D\left(a^{\prime}\right)} \in K\left(a^{D, n}: n>0\right)$ et que pour tout $m \geq n, a^{\prime m}$ est préfixe de $a_{\text {niv }\left(a^{m} / a^{n}\right)}^{\prime}$. De plus, par le maillage, on sait que $a^{\prime n}=\left(a_{1}^{\prime n}, \ldots, a_{k_{n}}^{\prime n}\right)$ avec $k_{n}$ suite strictement croissante (c.f. Remarque 4). Si la complexité de $a^{\prime}$ est 1 , on vérifie alors facilement que $a_{j}^{\prime n} \notin K\left\langle a_{j+1}^{\prime n}, \ldots, a_{k_{n}}^{\prime n}, a^{D\left(a^{\prime}\right)}\right\rangle$; pour cela il suffit de remarquer que quand on descend dans l'arbre, il reste toujours un degré de liberté à gauche. Cela suit du fait que pour tout $m \geq n$, l'uple extrait de $a^{m}$ correspondant à $a_{j}^{\prime n}$ par le lemme précédent est le point $a_{j}^{\prime}$. Ainsi $a^{\prime}$ a un arbre minoré sur $K\left\langle a^{D\left(a^{\prime}\right)}\right\rangle$. Si la complexité est $l^{\prime}+1$, alors par hypothèse d'induction, $a_{j}^{\prime n}$ a un arbre minoré sur $K\left\langle b^{D}\right\rangle$, où $b^{D}$ est le reste à droite de $a_{j}^{\prime n}$ dans $a^{n}$. Mais alors $b^{D}=a_{j+1}^{\prime n} \ldots a_{k_{n}}^{\prime n} a^{D, n}$ et $K\left\langle b^{D}\right\rangle \supseteq K\left\langle a^{D\left(a^{\prime}\right)}\right\rangle\left\langle a_{j+1}^{\prime n} \ldots a_{k_{n}}^{\prime n}\right\rangle$. D'où $a^{\prime}$ a un arbre minoré sur $K\left\langle a^{D\left(a^{\prime}\right)}\right\rangle$.

Nous ne savons pas si pour un ou tout $u$ de complexité $l$, le rang de Grill $u$ est majoré et si dans ce cas il vaut $\omega^{l}$

\section{Arbres majorés}

Afin de majorer le rang $\mathrm{U}$, nous allons compléter la construction de manière à ce que la déviation sur un modèle implique la réalisation d'une composante dans l'arbre. Dans un souci de simplification, nous utiliserons pour cela des arbres partiels «gauches». 
Définition 17. Un élément $a$ de $L$ a un arbre partiel gauche de niveau $m$ si $a \in \mathcal{B}_{m}$ et $a_{=m} \in K\left[a_{\overline{0}(m)}\right]$ où $a_{\overline{0}(m)}$ désigne le premier élément de l'uple $a_{=m}$. Dans ce cas $a=P\left(a_{\overline{0}(m)}\right)$ où $P$ est un polynôme de $K\left[X^{p^{m}}\right]$. Un tel polynôme qui définit un arbre partiel gauche de niveau $m$ sera appelé par la suite polynôme gauche de niveau $m$. Dans le cas où de plus tout élément de $a_{=m}$ est égal à la valeur d'un $p$-polynôme en $a_{\overline{0}(m)}$, on dira que l'arbre partiel gauche et le polynôme $P$ associé sont additifs.

Un élément $a$ de $L$ a un arbre gauche s'il a un arbre partiel gauche de niveau $m$ pour tout $m \geq 0$. Un type sur $K$ a un arbre gauche si toutes ces réalisations ont un arbre gauche.

Soient $u_{n}=v(u, n)$ et $s_{n}=s\left(u_{n}\right)$ pour tout $n<\omega$ (voir Section 2 pour les définitions de $v$ et $s$ ). Pour la suite, fixons $\beta(n)$ une entier positif et $P_{n, i}$ un polynôme gauche de niveau $\beta(n)$ pour tout $n<\omega$ et tout $1 \leq i \leq s_{n}$.

Définition 18. Soit $q_{u}$ le type sur $K$ défini par : a réalise $q_{u}$ s'il existe deux suites d'uples $a^{n}=\left(a_{1}^{n}, \ldots, a_{s_{n}}^{n}\right)$ et $b^{n}=\left(b_{1}^{n}, \ldots, b_{s_{n}}^{n}\right)$ de complexité $l$ et de décomposition $u_{n}$ telles que $a^{0}=a$ et pour tout $n$,

1. les éléments $a_{1}^{n}, \ldots, a_{s_{n}}^{n}$ sont algébriquement indépendants au-dessus de $K$;

2. $a_{i}^{n}=P_{n, i}\left(b_{i}^{n}\right)$ pour tout $1 \leq i \leq s_{n}$;

3. $M_{u_{n}}^{1}\left(b^{n}, a^{n+1}\right)$.

Remarque 19. - De même que pour $p_{u}$, les réalisations de $q_{u}$ ont un arbre minoré, et donc $\mathrm{RU}\left(q_{u}\right) \geq \omega^{l}$.

- Si les polynômes utilisés dans la définition de $q_{u}$ sont additifs alors $q_{u}$ est un type additif.

Par la suite, on choisira l'ensemble des polynômes gauches dans la définition de $q_{u}$ de façon à ce que $\operatorname{RU}\left(q_{u}\right) \leq \omega^{l}$. Cela sera vrai quand toute réalisation de $q_{u}$ aura un arbre fortement majoré.

Définition 20. On définit les notions d'arbre faiblement et fortement majoré par une induction imbriquée sur la complexité.

1. Un uple $a=\left(a_{1}, \ldots, a_{k}\right)$, de complexité 1 et de décomposition $k$, a un arbre faiblement majoré sur $K$ si pour tout $i \in\{1, \ldots, k\}, \operatorname{RT}\left(a / K\left\langle a_{i}\right\rangle\right)<k$. (Rappelons que $\mathrm{RT}\left(a / K\left\langle a_{i}\right\rangle\right)$ est le degré de transcendance de $K\left\langle a_{i} a\right\rangle$ sur $K\left\langle a_{i}\right\rangle$ et que RU $\leq$ $\mathrm{RT})$

2. Un uple $a$ de complexité $l$ a un arbre fortement majoré sur $K$ s'il existe une suite strictement croissante $\left(i_{n}\right)_{n<\omega}$ d'entiers positifs et une suite $\left(a^{n}\right)_{n<\omega}$ d'uples de complexité $l$ telles que

- $a^{n}$ a un arbre faiblement majoré sur $K$,

- $a^{n} \subset a_{=i_{n}}$ et $a \in K\left[a^{n}\right]$ pour tout $n$, et

- si le type de $a$ sur $K^{\prime}$ dévie sur $K$ pour une extension élémentaire $K^{\prime}$ de $K$, alors $a^{n} \cap K^{\prime} \neq \emptyset$ pour un entier $n$.

3. Un uple $a=\left(a_{1}, \ldots, a_{k}\right)$ de complexité $l+1$ a un arbre faiblement majoré sur $K$ si

- les uples $a_{i}$ de complexité $l$ ont tous un arbre faiblement majoré sur $K$, 
- l'uple $a_{i}$ (respectivement l'uple $a_{i+1}$ ) a un arbre fortement majoré sur $K\left\langle a_{i+1}\right\rangle$ (respectivement sur $K\left\langle a_{i}\right\rangle$ ).

Lemme 21. Si un uple a de complexitél a un arbre fortement majoré, alors $\mathrm{RU}(a / K) \leq$ $\omega^{l}$.

Démonstration. On montre par induction sur $l$ que si un uple $a$ de complexité $l$ et de décomposition $(k, v)$ a un arbre faiblement majoré sur $K$, alors $\operatorname{RU}(a / K\langle c\rangle)<k \omega^{l-1}$ pour tout $c \in a$, et que si un uple $a$ de complexité $l$ a un arbre fortement majoré sur $K$ alors $\mathrm{RU}(a / K) \leq \omega^{l}$.

Si $a$ est de complexité 1 et a un arbre faiblement majoré, alors par définiton $\mathrm{RU}(a / K\langle c\rangle) \leq \mathrm{RT}(a / K\langle c\rangle)<k$ pour tout $c \in a$.

Si $a$ est de complexité $l$ et a un arbre fortement majoré sur $K$. Soit $K^{\prime}$ une extension élémentaire de $K$ telle que le type de $a$ sur $K^{\prime}$ dévie sur $K$. Alors, il existe $a^{n}$ un uple de complexité $l$ tel que $a^{n}$ a un arbre faiblement majoré sur $K, a^{n}$ est interdéfinissable avec $a$ sur $K$, et $a^{n} \cap K^{\prime} \neq \emptyset$. D'où

$$
\operatorname{RU}\left(a / K^{\prime}\right)=\operatorname{RU}\left(a^{n} / K^{\prime}\right) \leq \operatorname{RU}\left(a^{n} / K\left\langle a^{n} \cap K^{\prime}\right\rangle\right)<\omega^{l} .
$$

Par conséquent $\operatorname{RU}(a / K) \leq \omega^{l}$.

Si $a=\left(a_{1}, \ldots, a_{k}\right)$ est de complexité $l+1$ et a un arbre faiblement majoré sur $K$. Alors pour tout $c \in a$, il existe $i \in\{1, \ldots, k\}$ tel que $c \in a_{i}$. Par conséquent,

$$
\mathrm{RU}(a / K\langle c\rangle) \leq \bigoplus_{j<i} \mathrm{RU}\left(a_{j} / K\left\langle a_{j+1}\right\rangle\right) \oplus \bigoplus_{j>i} \mathrm{RU}\left(a_{j} / K\left\langle a_{j-1}\right\rangle\right) \oplus \mathrm{RU}\left(a_{i} / K\langle c\rangle\right) .
$$

Pour $j<i$, l'uple $a_{j}$ de complexité $l$ a un arbre fortement majoré sur $K\left\langle a_{j+1}\right\rangle$ et par induction $\mathrm{RU}\left(a_{j} / K\left\langle a_{j+1}\right\rangle\right) \leq \omega^{l}$. De même pour $j>i, \operatorname{RU}\left(a_{j} / K\left\langle a_{j-1}\right\rangle\right) \leq \omega^{l}$. Enfin l'uple $a_{i}$ de complexité $l$ a un arbre faiblement majoré sur $K$, donc $\operatorname{RU}\left(a_{i} / K\langle c\rangle\right)<\omega^{l}$. D'où $\operatorname{RU}(a / K\langle c\rangle)<k \omega^{l}$.

Dans la section précédente nous avons introduit la notion d'uples extraits (voir Définition 14). Dans la suite nous utiliserons une notion d'uples extraits adjacents.

Définition 22. Soit $a=\left(a_{1}, \ldots, a_{k}\right)$ un $\left(k, u^{\prime}\right)$-uple où les $a_{i}$ sont des $u^{\prime}$-uples (ou des éléments si la complexité de $a$ vaut 1$)$. Deux uples $x_{1}$ et $x_{2}$ extraits de $a$ sont adjacents si $\left(x_{1}, x_{2}\right)=\left(a_{i}, a_{i+1}\right)$ pour un $i<k$ ou si $\left(x_{1}, x_{2}\right)$ forme un couple d'uples extraits adjacents d'un même $a_{i}$ pour un $i \leq k$.

Par une preuve analogue au Lemme 15 on vérifie le résultat suivant.

Lemme 23. Soient deux uples a et b vérifiant $M_{u}^{n}(a, b)$ pour un entier $n \geq 1$ et la décomposition $u$ de $a$. Soit $x$ le premier élément d'un couple $\left(x, x^{\prime}\right)$ d'uples extraits adjacents de a de complexité $l^{\prime}$. Soit y l'unique uple extrait de b de même complexité $l^{\prime}$ qui soit un préfixe de $x_{\operatorname{niv}(b / a)}$. Alors

$$
a_{\operatorname{niv}(b / a)}^{D(x)}=b^{D(y)}
$$

et, de manière équivalente (par la Propriété 11 et le Lemme 12), si $x=\left(x_{k}, \ldots, x_{l}\right)$ est le sous-uple d'éléments consécutifs de l'uple $a=\left(x_{1}, \ldots, x_{s(u)}\right)$, alors $y=\left(y_{s}, \ldots, y_{t}\right)$ est le sous-uple d'éléments consécutifs de l'uple $b=\left(y_{1}, \ldots, y_{s(v(u, n))}\right)$, avec $s=\min C_{u, k}^{n}$ et $t=\max \left(C_{u, l}^{n} \backslash C_{u, l+1}^{n}\right)$. 
Hypothèse 24. Si on a $1 \leq i \leq j \leq s_{n}$ et $P \in K\left[X_{i}, \ldots, X_{j}, \bar{Y}\right]$, a une réalisation de $q_{u}$ et $c$ un uple de $L$ tel que $P\left(a_{i}^{n}, \ldots, a_{j}^{n}, c\right)=0$ et $P\left(X_{i}, . ., X_{j}, c\right) \neq 0$, alors ils existent

- un entier $m \geq n$;

- un indice $k \in \bigcup_{i \leq r \leq j} C_{u_{n}, r}^{m-n}$

- si $j<s_{n}$, un indice $k_{g} \in \bigcup_{i<r<j} C_{u_{n}, r}^{m-n} \backslash C_{u_{n}, j+1}^{m-n}$;

- si $i>1$, un indice $k_{d} \in \bigcup_{i \leq r \leq j} C_{u_{n}, r}^{m-n} \backslash C_{u_{n}, i-1}^{m-n}$

tels que

- l'uple $a_{k}^{m} \in K\langle c\rangle$;

- si $j<s_{n}$, l'uple $a_{k_{g}}^{m} \in K\left\langle c, a_{j+1}^{n}\right\rangle$;

- si $i>1$, l'uple $a_{k_{d}}^{m} \in K\left\langle c, a_{i-1}^{n}\right\rangle$.

Définition 25. Soit $a$ une réalisation de $q_{u}$ et considérons la suite d'uples $a^{n}$ donnée dans la Définition 18. Soit $n \leq m$. De manière analogue à la Définition 13 , si $x=$ $\left(x_{k}, \ldots, x_{l}\right)$ est un sous-uple d'éléments consécutifs de l'uple $a_{n}=\left(x_{1}, \ldots, x_{s\left(u_{n}\right)}\right)$, on note $x_{\text {niv }(m)}$ le sous-uple $\left(y_{s}, \ldots, y_{t}\right)$ d'éléments consécutifs de l'uple $a_{m}=\left(y_{1}, \ldots, y_{s\left(u_{m}\right)}\right)$, avec $s=\min C_{u_{n}, k}^{m-n}$ et $t=\max C_{u_{n}, l}^{m-n}$.

Dans le cas où $x$ est un uple extrait de $a_{n}$, on note $x^{g, m}$ l'unique préfixe de $x_{\text {niv }(m)}$ qui soit un uple extrait de $a_{m}$ de même complexité. (Notons ici que les hyptohèses ne sont pas exactement celles du Lemme 15 mais que de manière évidente on obtient les propriétés correspondantes dans ce cadre). On note symétriquement $x^{d, m}$ l'unique suffixe de $x_{\text {niv }(m)}$ qui soit un uple extrait de $a_{m}$ de même complexité.

Proposition 26. Si $q_{u}$ satisfait l'Hypothèse 24 alors $\mathrm{RU}\left(q_{u}\right)=\omega^{l}$.

Démonstration. Par la remarque 19 et le lemme 21, il suffit de montrer que toute réalisation de $q_{u}$ a un arbre fortement majoré sur $K$.

Soit $a$ une réalisation de $q_{u}$ et $\left(a^{n}\right)_{n \geq 0}$ la suite d'uples $a^{n}$ donnée dans la définition 18 .

Soient $x_{1}$ et $x_{2}$ deux uples adjacents extraits de $a^{n}$. Par les propriétés correspondantes dans ce cadre à celles obtenues dans le Lemme 23 , on a pour tout $m \geq n$,

et donc

- $\left(x_{1}\right)_{\operatorname{niv}(m)} \subseteq x_{1}^{g, m} x_{2 \operatorname{niv}(m)} ;$

$-\left(x_{2}\right)_{\operatorname{niv}(m)} \subseteq x_{1 \operatorname{niv}(m)} x_{2}^{d, m} ;$

$-\left(x_{1}\right)_{\operatorname{niv}(m)} \in K\left\langle x_{2}\right\rangle\left[x_{1}^{g, m}\right]$;

- $\left(x_{2}\right)_{\operatorname{niv}(m)} \in K\left\langle x_{1}\right\rangle\left[x_{2}^{d, m}\right]$.

Supposons de plus que le type de $x_{1}$ sur $K^{\prime}$ dévie sur $K\left\langle x_{2}\right\rangle$ (où $K^{\prime}$ est une extension élémentaire de $\left.K\left\langle x_{2}\right\rangle\right)$. Alors il existe $m \geq n, c \in K^{\prime}$ et $P \in K[\bar{X}, \bar{Y}]$, tels que $P\left(x_{1}^{g, m}, c\right)=0$ et $P(\bar{X}, c) \neq 0$. En utilisant l'Hypothèse 24 (et le Lemme 23), on en déduit qu'il existe $m^{\prime} \geq m$ tel que $x_{1}^{g, m^{\prime}} \cap K\left\langle c, x_{2}\right\rangle \neq \emptyset$. D'où $x_{1}^{g, m^{\prime}} \cap K^{\prime} \neq \emptyset$.

Montrons alors par induction sur la complexité que si $x$ est un uple extrait d'un $a^{n}$ alors $x$ a un arbre faiblement majoré sur $K$ et que si $x_{1}$ et $x_{2}$ sont deux uples adjacents extraits d'un $a^{n}$ alors $x_{1}$ a un arbre fortement majoré sur $K\left\langle x_{2}\right\rangle$ et $x_{2}$ a un arbre fortement majoré sur $K\left\langle x_{1}\right\rangle$ : 
1. Soit $x=\left(e_{1}, . ., e_{r}\right)$ un uple de complexité 1 extrait d'un $a^{n}$. Soit $i \in\{1, \ldots r\}$. Pour tout $m \geq n$, on a alors par le Lemme 23

$$
x_{\mathrm{niv}(m)} \subseteq e_{1}^{g, m} \ldots e_{i-1}^{g, m}\left(e_{i}\right)_{\operatorname{niv}(m)} e_{i+1}^{d, m} \ldots e_{r}^{d, m} .
$$

Donc

$$
x_{\mathrm{niv}(m)} \in K\left\langle e_{i}\right\rangle\left[e_{1}^{g, m}, \ldots, e_{i-1}^{g, m}, e_{i+1}^{d, m}, \ldots, e_{r}^{d, m}\right] .
$$

On en déduit que $\operatorname{RT}\left(x / K\left\langle e_{i}\right\rangle\right)<r$ et donc que $x$ est faiblement majoré sur $K$.

2. Supposons que tout uple de complexité $l$ extrait d'un $a^{n}$ est faiblement majoré sur $K$. Soit $\left(x_{1}, x_{2}\right)$ couple d'uples adjacents extraits d'un $a^{n}$ et de complexité $l$. Par hypothèse d'induction, l'uple $x_{1}^{g, m}$ est faiblement majoré sur $K$ et par une des remarques précédentes, on a $x_{1} \in K\left\langle x_{2}\right\rangle\left[x_{1}^{g, m}\right]$, pour tout $m$. Enfin, on a également remarqué que si le type de $x_{1}$ sur $K^{\prime}$ dévie sur $K\left\langle x_{2}\right\rangle$ alors il existe $m$ tel que $x_{1}^{g, m} \cap K^{\prime} \neq \emptyset$. Par conséquent, $x_{1}$ est fortement majoré sur $K\left\langle x_{2}\right\rangle$. On vérifie symétriquement que $x_{2}$ est fortement majoré sur $K\left\langle x_{1}\right\rangle$.

3. Supposons le résultat vrai pour les uples extraits de complexité $l$. Soit $x$ un uple de complexité $l+1$ extrait d'un $a^{n}$. Alors $x=\left(e_{1}, . ., e_{r}\right)$ où chaque $e_{i}$ est un uple de complexité $l$, également extrait de $a^{n}$. Par hypothèse d'induction, chaque $e_{i}$ a un arbre faiblement majoré sur $K$, fortement majoré sur $K\left\langle e_{i+1}\right\rangle$ et $e_{i+1}$ a un arbre fortement majoré sur $K\left\langle e_{i}\right\rangle$. Par conséquent $x$ a un arbre faiblement majoré sur $K$.

On en déduit que chaque $a^{n}$ est faiblement majoré sur $K$. De plus par Hypothèse 24, si le type de $a$ sur $K^{\prime}$ dévie sur $K$, il existe $n$ tel que $a^{n} \cap K^{\prime} \neq \emptyset$. Par conséquent $a$ a un arbre fortement majoré sur $K$.

Pour satisfaire l'Hypothèse 24, on procède de la même façon que pour la construction de types de rang $\omega$ dans [1].

Fixons $K_{0}$ une sous-structure élémentaire dénombrable de $L$ contenant $b$.

Proposition 27. A partir d'une infinité de types minimaux sur $K_{0}$, deux à deux orthogonaux et qui ont tous un arbre gauche, on construit un type $q_{u}$ sur $K_{0}$ qui satisfait l'Hypothèse 24.

Démonstration. Soit $\left(p_{i}\right)_{i>0}$ une famille de types minimaux deux à deux orthogonaux ayant un arbre gauche. Par un simple argument de compacité [1, Lemma 5.4], pour tout polynôme $Q \in K_{0}\left[X_{1}, \ldots, X_{n}, \bar{Y}\right]$, il existe $m(Q)<\omega$ tel que pour tout $\left(c_{j}\right)_{j \in\{1, \ldots, n\}} \in$ $L$, tout uple $d \in L$ et tout $I \subseteq\{1, \ldots, n\}$ :

$$
\begin{gathered}
\bigwedge_{i \in I} p_{i, \leq m(Q)}\left(x_{i}\right) \vdash\left(Q\left(\left(X_{i}\right)_{i \in I},\left(c_{j}\right)_{j \notin I}, d\right) \neq 0 \bigwedge Q\left(\left(x_{i}\right)_{i \in I},\left(c_{j}\right)_{j \notin I}, d\right)=0\right) \\
\Rightarrow \bigvee_{i \in I} " x_{i} \in K_{0}\left\langle d, c_{j}: j \notin I\right\rangle "
\end{gathered}
$$

(Pour un type $p$, la notation $p_{\leq m(Q)}(x)$ représente l'ensemble des équations et des inéquations satisfaites par les composantes de niveau inférieur ou égal à $n$ d'une réalisation de $p$, voir [1, Notation 5.3].) 
Soient $\bar{Y}$ une uple infini dénombrable d'indéterminées et $\left(Q_{n}\right)_{n \in \omega}$ une énumération des polynômes dans

$$
\cup_{n \in \omega} K_{0}\left[X_{1, n}, \ldots, X_{s_{n}, n}, \bar{Y}\right]
$$

telle que pour tout $n<\omega$,

$$
Q_{n} \in \cup_{m \leq n} K_{0}\left[X_{1, m}, \ldots, X_{s_{m}, m}, \bar{Y}\right] .
$$

On définit alors par induction sur $n$, les polynômes $P_{n, i}$ des équations $a_{i}^{n}=P_{n, i}\left(b_{i}^{n}\right)$ dans l'arbre $q_{u}$. Supposons les polynômes $P_{m, i}$ définis pour tout $m<n$. Soit $m$ tel que $Q_{n} \in K_{0}\left[X_{1, m}, \ldots, X_{s_{m}, m}, \bar{Y}\right]$. Il existe pour tout $i \in\left\{1, \ldots, s_{m}\right\}$, un polynôme $R_{i} \in K_{0}\left[X_{j}: j \in C_{u_{m}, i}^{n-m}\right]$ tel que $a_{i}^{m}=R_{i}\left(a_{j}^{n}: j \in C_{u_{m}, i}^{n-m}\right)$. Soit

$$
S_{n}\left(X_{1, n}, \ldots, X_{s_{n}, n}, \bar{Y}\right)=Q_{n}\left(R_{1}\left(X_{1, n}, \ldots, X_{s_{n}, n}\right), \ldots, R_{s_{m}}\left(X_{1, n}, \ldots, X_{s_{n}, n}\right), \bar{Y}\right) .
$$

Alors pour tout $i \in\left\{1, \ldots, s_{n}\right\}$, on définit $P_{n, i}$ de façon que $a_{i}^{n}$ satisfasse $p_{i, \leq m\left(S_{n}\right)}$.

Vérifions qu'alors $q_{u}$ satisfait l'Hypothèse 24 . Soient $1 \leq i \leq j \leq s_{m}, Q \in K_{0}\left[X_{i}, \ldots, X_{j}, \bar{Y}\right]$, $a$ une réalisation de $q_{u}$ et $c$ un uple de $L$ tels que $Q\left(a_{i}^{m}, \ldots, a_{j}^{m}, c\right)=0$ et $Q\left(X_{i}, \ldots, X_{j}, c\right) \neq$ 0 . Considérons le polynôme $Q_{n} \in K_{0}\left[X_{i, m}, \ldots, X_{j, m}, \bar{Y}\right] \subseteq K_{0}\left[X_{1, m}, \ldots, X_{s_{m}, m}, \bar{Y}\right]$ correspondant à $Q$ dans l'énumération. Alors

$$
\begin{gathered}
S_{n}\left(a_{k}^{n}, c: k \in \cup_{i \leq r \leq j} C_{u_{m}, r}^{n-m}\right)=0, \\
S_{n}\left(X_{k, n}, c: k \in \cup_{i \leq r \leq j} C_{u_{m}, r}^{n-m}\right) \neq 0, \\
\text { si } j<s_{m}, S_{n}\left(X_{k, n}, a_{l}^{n}, c: k \in \cup_{i \leq r \leq j} C_{u_{m}, r}^{n-m} \backslash C_{u_{m}, j+1}^{n-m}, l \in C_{u_{m}, j+1}^{n-m}\right) \neq 0,
\end{gathered}
$$

et

$$
\text { si } i>0, S_{n}\left(X_{k, n}, a_{l}^{n}, c: k \in \cup_{i \leq r \leq j} C_{u_{m}, r}^{n-m} \backslash C_{u_{m}, i-1}^{n-m}, l \in C_{u_{m}, i-1}^{n-m}\right) \neq 0 .
$$

(Les deux dernières inéquations découlent du fait que $Q_{n}\left(X_{i}, \ldots, X_{j}, c\right) \neq 0$ et du Lemme 12.)

Par conséquent, il existe $k \in \cup_{i \leq r \leq j} C_{u_{m}, r}^{n-m}, k_{g} \in \cup_{i \leq r \leq j} C_{u_{m}, r}^{n-m} \backslash C_{u_{m}, j+1}^{n-m}$ et $k_{d} \in$ $\cup_{i \leq r \leq j} C_{u_{m}, r}^{n-m} \backslash C_{u_{m}, i-1}^{n-m}$ tels que $a_{k}^{n} \in K_{0}\langle c\rangle, a_{k_{g}}^{n} \in K_{0}\left\langle c, a_{l}^{n}: l \in C_{u_{m}, i-1}^{n-m}\right\rangle \subset K_{0}\left\langle c, a_{j+1}^{m}\right\rangle$, et $a_{k_{d}}^{n} \in K_{0}\left\langle c, a_{l}^{n}: l \in C_{u_{m}, i-1}^{n-m}\right\rangle \subset K_{0}\left\langle c, a_{i-1}^{m}\right\rangle$.

Corollaire 28. Pour tout ordinal $\lambda<\omega^{\omega}$, il existe un sous-groupe additif défini sur $K_{0}$, non mono-basé et de rang $U$ égal à $\lambda$.

Démonstration. Il suffit de montrer le résultat pour tout rang $\omega^{l}$ : en effet, à partir de tels groupes on obtient tous les rangs $\lambda<\omega^{\omega}$ en considérant des produits cartésiens et en utilisant le fait que pour tout entier $n>0$, le groupe $L^{\times p^{\nu n}}$ est définissablement isomorphe avec $L$.

Pour $l=0$, on a $L^{p^{\infty}}$ qui est de rang 1 et non monobasé.

Pour $l>0$, on considère une décomposition $u$ de complexité $l$. D'après [3], on peut considérer une famille $\left(p_{i}\right)_{i>0}$ de types additifs minimaux définis sur $K_{0}$, deux à deux orthogonaux et ayant un arbre gauche. D'après la proposition précédente on obtient un type $q_{u}$ de rang $\omega^{l}$. Du fait que les types $p_{i}$ sont additifs, on en déduit que $q_{u}$ est le générique d'un groupe additif $G_{u}$. 
En utilisant les notations de la Propriété 5, on vérifie par induction que si $a=$ $\left(x^{p^{m_{u, n}}}, 0, \ldots, 0\right)$ et $b=(x, 0, \ldots, 0)$ sont des uples de décomposition $u$ et $v(u, n)$ respectivement dont le seul élément non nul est $x^{p^{m_{u, n}}}$ et $x$ respectivement, alors on a le maillage $M_{u}^{n}(a, b)$. On en déduit que si $a$ est un uple de décomposition $u$ dont le seul élément non nul est son premier élément qui de plus appartient à $L^{p^{\infty}}$ alors $a$ satisfait les systèmes $S_{u, n}$. Le groupe $L^{p^{\infty}}$ se plonge donc dans Grill ${ }_{u}$. Si on suppose par ailleurs que $p_{1}$ est le type générique de $L^{p^{\infty}}$, on en déduit alors que $L^{p^{\infty}}$ se plonge dans $G_{u}$ et donc que $G_{u}$ n'est pas monobasé.

Complétons maintenant ces constructions afin d'obtenir des groupes monobasés :

Hypothèse 29. Le type $q_{u}$ est additif, défini sur $K_{0}$, et si $G_{u}$ est le groupe additif associé à $q_{u}$ alors pour tous entiers $k$ et $n$ et tout polynôme $P \in K_{0}\left[\bar{X}^{1, n}, \ldots, \bar{X}^{k, n}, \bar{Y}\right]$, il existe un entier $m \geq n$ et un polynôme $Q \in K_{0}\left[\bar{X}^{1, m}, \ldots, \bar{X}^{k, m}, \bar{Y}\right]$ tel que :

1. Pour tout $\left(a^{1}, \ldots, a^{k}\right) \in G_{u}^{\times k}$ et $c \in L, P\left(a^{1, n}, \ldots, a^{k, n}, c\right)=0$ si et seulement si $Q\left(a^{1, m}, \ldots, a^{k, m}, c\right)=0$.

2. Pour toutes parties $J_{1}, \ldots, J_{s_{m}}$ de $\{1, \ldots, k\}$ et tous $\lambda_{i, j, j^{\prime}} \in \mathbb{F}_{p}$ où $i \in\left\{1, \ldots, s_{m}\right\}$, $j \in\{1, \ldots, k\} \backslash J_{i}$ et $j^{\prime} \in J_{i}$, considérons $R \in K_{0}\left[\bar{Z}^{1, m}, \ldots, \bar{Z}^{k, m}, \bar{Y}\right]$ le polynôme $Q$ où l'on a remplacé $X_{i}^{j, m} \operatorname{par} Z_{i}^{j, m}$ si $j \in J_{i}$, et par $Z_{i}^{j, m}+\sum_{j^{\prime} \in J_{i}} \lambda_{i, j, j^{\prime}} Z_{i}^{j^{\prime}, m}$ sinon. Soient $\left(a^{1}, \ldots, a^{k}\right) \in G_{u}^{\times k},\left(c_{i}^{j}\right)_{i \in\left\{1, \ldots, s_{m}\right\}, j \notin J_{i}}$ dans $L$ et $c \in L$ tels que

- $R\left(\left(a_{i}^{j, m}\right)_{i \in\left\{1, \ldots, s_{m}\right\}, j \in J_{i}},\left(c_{i}^{j}\right)_{i \in\left\{1, \ldots, s_{m}\right\}, j \notin J_{i}}, c\right)=0$ et

- $R\left(\left(Z_{i}^{j, m}\right)_{i \in\left\{1, \ldots, s_{m}\right\}, j \in J_{i}},\left(c_{i}^{j}\right)_{i \in\left\{1, \ldots, s_{m}\right\}, j \notin J_{i}}, c\right) \neq 0$

alors il existe $i_{0} \in\left\{1, \ldots, s_{m}\right\}$ tel qu'une combinaison linéaire non triviale sur $\mathbb{F}_{p}$ des $\left(a_{i_{0}}^{j, m}\right)_{j \in J_{i_{0}}}$ appartient à $K_{0}\left\langle c, c_{i}^{j}: i \in\left\{1, \ldots, s_{m}\right\}, j \notin J_{i}\right\rangle$.

Proposition 30. Si $q_{u}$ satisfait les Hypothèses 24 et 29 alors $G_{u}$ est un groupe monobasé de rang $\omega^{l}$.

Démonstration. On doit montrer que $G_{u}$ est mono-basé. Pour cela, on vérifie le lemme suivant :

Lemme 31. Soient $K_{1}$ une sous-structure élémentaire de $L$ et $\left(a^{1}, \ldots, a^{k}\right) \in G_{u}^{\times k}$. Le type de $\left(a^{1}, \ldots, a^{k}\right)$ sur $K_{1}$ est déterminé par la connaissance, pour chaque entier $n$ et chaque $i \in\left\{1, \ldots, s_{n}\right\}$ des combinaison linéaires des $a_{i}^{1, n}, \ldots, a_{i}^{k, n}$ sur $\mathbb{F}_{p}$ qui sont dans $K_{1}$.

Preuve du lemme. Soient $\left(a^{1}, \ldots, a^{k}\right) \in G_{u}^{\times k}$ et $\left(b^{1}, \ldots, b^{k}\right) \in G_{u}^{\times k}$ tels que pour chaque entier $n$, chaque $i \in\left\{1, \ldots, s_{n}\right\}$ et tous $\lambda_{1}, \ldots, \lambda_{k} \in \mathbb{F}_{p}$,

$$
\sum_{j=1}^{k} \lambda_{j} a_{i}^{j, n} \in K_{1} \text { si et seulement si } \sum_{j=1}^{k} \lambda_{j} b_{i}^{j, n} \in K_{1},
$$

et si $\sum_{j=1}^{k} \lambda_{j} a_{i}^{j, n} \in K_{1}$ alors

$$
\sum_{j=1}^{k} \lambda_{j} a_{i}^{j, n}=\sum_{j=1}^{k} \lambda_{j} b_{i}^{j, n}
$$


Supposons que $\left(a^{1}, \ldots, a^{k}\right)$ et $\left(b^{1}, \ldots, b^{k}\right)$ n'ont pas même type sur $K_{1}$. Alors on peut supposer qu'il existe $n$ un entier, $c \in K_{1}$ et $P \in K_{0}\left[\bar{X}^{1, n}, \ldots, \bar{X}^{k, n}, \bar{Y}\right]$ tels que

$$
P\left(a^{1, n}, \ldots, a^{k, n}, c\right)=0 \text { et } P\left(b^{1, n}, \ldots, b^{k, n}, c\right) \neq 0 .
$$

Soient $m$ et $Q$ associé à $n$ et $P$ dans l'Hypothèse 29. Par hypothèse, pour tout $i \in$ $\left\{1, \ldots, s_{m}\right\}$ il existe une partie $J_{i}$ de $\{1, . ., k\}$ tel que $\left(a_{i}^{j, m}\right)_{j \in J_{i}}$ et $\left(b_{i}^{j, m}\right)_{j \in J_{i}}$ sont respectivement des bases de $\mathbb{F}_{p^{-}}$-espaces vectoriels engendrés par les $a_{i}^{j, m}$ et les $b_{i}^{j, m}$ au-dessus de $K_{1}$. Alors pour tout $i$ et tout $j \notin J_{i}$, il existe des $\lambda_{i, j, j^{\prime}} \in \mathbb{F}_{p}$ tels que

$$
a_{i}^{j, n}-\sum_{j^{\prime} \in J_{i}} \lambda_{i, j, j^{\prime}} a_{i}^{j^{\prime}, n}=b_{i}^{j, n}-\sum_{j^{\prime} \in J_{i}} \lambda_{i, j, j^{\prime}} b_{i}^{j^{\prime}, n}=c_{i}^{j} \in K_{1} .
$$

Maintenant utilisons le polynôme $R$ correspondant dans l'Hypothèse 29. Alors

$$
R\left(\left(a_{i}^{j, m}\right)_{i \in\left\{1, \ldots, s_{m}\right\}, j \in J_{i}},\left(c_{i}^{j}\right)_{i \in\left\{1, \ldots, s_{m}\right\}, j \notin J_{i}}, c\right)=0
$$

car $P\left(a^{1, n}, \ldots, a^{k, n}, c\right)=0$ et donc $Q\left(a^{1, m}, \ldots, a^{k, m}, c\right)=0$.

Par ailleurs comme $P\left(b^{1, n}, \ldots, b^{k, n}, c\right) \neq 0$ on a $Q\left(b^{1, m}, \ldots, b^{k, m}, c\right) \neq 0$ et donc

$$
R\left(\left(Z_{i}^{j, m}\right)_{i \in\left\{1, \ldots, s_{m}\right\}, j \in J_{i}},\left(c_{i}^{j}\right)_{i \in\left\{1, \ldots, s_{m}\right\}, j \notin J_{i}}, c\right) \neq 0 .
$$

On en déduit par l'Hypothèse 29 qu'il existe $i \in\left\{1, \ldots, s_{m}\right\}$ tel que $\left(a_{i}^{j, m}\right)_{j \in J_{i}}$ n'est pas libre au-dessus de $K_{1}$, ce qui est en contradiction avec le choix de $J_{i}$.

En utilisant ce lemme, il est alors facile de vérifier que la base canonique du type de $\left(a^{1}, \ldots, a^{k}\right)$ sur $K_{1}$ est incluse dans

$$
K_{0}\left\langle\sum_{j=1}^{k} \lambda_{j} a_{i}^{j, n}: n<\omega, i \in\left\{1, . ., s_{n}\right\}, \lambda_{1}, \ldots, \lambda_{k} \in \mathbb{F}_{p}\right\rangle .
$$

Par conséquent ce type est monobasé.

Remarque. La géométrie du type $q_{u}$ est la suivante : des réalisations $a^{1}, \ldots, a^{k}$ sont indépendantes au-dessus de $K$ si et seulement si pour tout $n$ et tout $i \leq s_{n}$, les composantes $a_{i}^{1, n}, \ldots, a_{i}^{k, n}$ sont $\mathbb{F}_{p}$-linéairement indépendantes au-dessus de $K$.

Corollaire 32. Pour toute famille dénombrable $\left(\lambda_{i}\right)_{i \in \omega}$ d'ordinaux strictement inférieurs à $\omega^{\omega}$, il existe une famille de sous-groupes additifs monobasés $G_{i}$ deux à deux orthogonaux et de rang $U$ respectif égal à $\lambda_{i}$.

Démonstration. Notons qu'il suffit de montrer qu'il existe une famille de types sur $K_{0}$ qui sont additifs, monobasés, deux à deux orthogonaux et tels que pour tout $l<\omega$, une infinité d'entre eux ont un rang $\omega^{l}$. Pour cela, on utilise une famille $\left(p_{i}\right)_{i>0}$ de types additifs minimaux définis sur $K_{0}$, deux à deux orthogonaux, ayant tous un arbre gauche et tels que les sous-groupes additifs associés ont une structure induite réduite à un $\mathbb{F}_{p}$-espace vectoriel. L'existence d'une telle famille est montrée dans [1, Corollary 4.20]. 
De manière analogue à la preuve de [1, Corollary 5.6], pour toute décomposition $u$, on peut construire avec cette famille un type $q_{u}$ satisfaisant les Hypothèses 24 et 29 . On a alors un type additif de rang $\omega^{l}$ et monobasé.

De plus, en partitionnant notre famille $p_{i}$ en une infinité de parties infinis, on peut construire simultanément une famille infinie $\left(q^{t}\right)_{t<\omega}$ de tels types de décompositions respectives $u^{t}$ et qui sont de plus deux à deux orthogonaux : on peut en effet considérer à chaque étape d'induction un polynôme portant sur une partie des types en construction.

Nous venons de donner des constructions de types additifs monobasés, par contre nous ne savons pas s'il est possible de construire des types additifs localement modulaires non monobasés.

\section{Références}

[1] T. Blossier, Subgroups of the additive group of a separably closed field, A.P.A.L. (134), 169-216, 2005.

[2] T. Blossier, K. Krupiński, A special thin type, Illinois J. Math. 49, no. 1, 281-290, 2005.

[3] E. Bouscaren, F. Delon, Minimal groups in separably closed fields, J. Symb. Logic (67), No. 1, 239-259, 2002.

[4] F. Delon, Idéaux et types sur les corps séparablement clos, Supp. au Bull. de la S.M.F., Mémoire 33, Tome 116, 1998.

[5] F. Delon, Separably closed fields, Model theory and algebraic geometry, E. Bouscaren (Ed.), Springer-Verlag, Berlin, 143-176, 1998.

[6] Z. Chatzidakis, G. Cherlin, S. Shelah, G. Srour, C. Wood, Orthogonality of types in separably closed fields, Classification theory, Proceedings of the 1985 Chicago conference, Lecture Notes in Mathematics, vol. 1292, Springer-Verlag.

[7] Z. Chatzidakis, C. Wood, Minimal types in separably closed fields, J. Symb. Logic (65), No. 3, 1443-1450, 2000.

[8] Y. Ershov, Fields with a solvable theory, Doklady, vol. 174, 19-20, 1967; English translation in Soviet Mathematics 8, 575-576, 1967.

[9] D. Ghioca, The Mordell-Lang theorem for Drinfeld modules, Int. Math. Res. Not., no. 53, 3273-3307, 2005.

[10] E. Hrushovski, The Mordell-Lang conjecture for function fields, J. Amer. Math. Soc. (9), no. 3, 667-690, 1996.

Université de Lyon, Université Lyon 1, Institut Camille Jordan UMR 5208 CNRS, 43 boulevard du 11 novembre 1918, F-69622 Villeurbanne Cedex, France

E-mail : blossier@math.univ-lyon1.fr 